\title{
Refractive Results after Cataract Surgery Using Optical Biometry
}

\author{
Mehvash Hussain ${ }^{1}$, Muhammad Muneer Quraishy ${ }^{2}$, Muhammad Akram ${ }^{3}$ \\ ${ }^{1,2}$ Department of Ophthalmology, Dow Medical College, ${ }^{3}$ Ruth K M Pfau Civil Hospital, Karachi
}

\begin{abstract}
Purpose: To assess the refractive outcome of optical biometry (Nidek AL-scan) after elective phacoemulsification in a study of 30 eyes.

Study Design: Descriptive case series.

Place and Duration of Study: Elective cataract surgeries done at a private clinic from July 2015 to June 2016 were selected and their records were analyzed.

Material and Methods: The measurements of IOL calculation was done using optical biometry with partial coherence interferometry (Nidek AL-scan) that provides information about axial length, central keratometry, white to white diameter and anterior chamber anatomical depth. SRK-T formula was used to calculate IOL power. All patients underwent a complete ophthalmological examination. Phacoemulsification with clear corneal incision of $2.75 \mathrm{~mm}$ was done and IOL was implanted in the bag (Alcon Acrysof SN60WF IOL and MA60AC IOL). Postoperative refraction was taken with autorefractor (Huvitz HRK-7000) after 4 weeks and it was compared with preoperative objective refraction. Comparison of $\mathrm{K}$ readings taken by AL-scan and autorefractor were done.

Results: We studied 30 eyes of 23 patients who underwent elective cataract surgery with foldable IOL. Postoperative spherical equivalent was Plano in $53 \%$ of cases with mean of -0.05 after 4 weeks postoperatively. The mean keratometric power using autorefractor was 44.4 D while with AL-scan it was 44.7 D. There were no intraoperative complications or postoperative subjective complaints (such as halo or glare) in our patients.

Conclusion: Intraocular lens power calculations done by optical biometry are easy to use, reliable and result in excellent refractive outcomes. Ultrasound biometry may still be required in case of mature and dense posterior subcapsular cataract.
\end{abstract}

Key Words: Biometry, optical biometry, phacoemulsification, cataract.

How to Cite this Article: Hussain M, Quraishy MM, Akram M. Refractive Results after Cataract Surgery Using Optical Biometry, Pak J Ophthalmol. 2020; 36 (1): 53-56.

DOI: https://doi.org/10.36351/pjo.v36i1.994

\section{INTRODUCTION}

Most clinicians now use optical biometry for intraocular lens (IOL) power calculations in their practice. Optical biometry is a highly accurate noninvasive automated method for measuring the anatomical characteristics of the eye.

Correspondence to: Mehvash Hussain

Assistant Professor of Ophthalmology

Dow Medical College

Email: mehvashh@hotmail.com
Accurate measurements are critical for determining the correct power of an IOL before it is implanted during cataract surgery ${ }^{1}$. The process of measuring the various anatomical characteristics of the eye that are needed for IOL power calculation is called ocular biometry. Optical biometry with the AL scan uses partial coherence interferometry to calculate IOL power. It is highly accurate, easy to perform, noninvasive method and is comfortable for the patient.

Third-generation formulae, such as the SRK/T and Hoffer Q use the axial length (AL) and keratometry 
(K) values to predict IOL power ${ }^{2,3}$. Precise measurements of keratometric data and axial length are very important. The optical biometric method is more precise and reproducible compared to ultrasound measurement ${ }^{4,5}$.

The advantages of optical compared with USB method are that it is easy to use, has reduced risks of trauma and infection, and is comfortable for the patient as well ${ }^{6,7}$. However, optical biometry is not accurate in eyes with mature or dense posterior sub-capsular cataracts and in certain macular diseases ${ }^{8}$.

The purpose of the study was to find the refractive outcomes of optical biometry (Nidek AL-scan) after elective phacoemulsification.

\section{MATERIAL AND METHODS}

Patients who underwent cataract surgery with foldable IOL (Alcon Acrysof SN60WF IOL and MA60AC IOL) from $1^{\text {st }}$ July 2015 to $30^{\text {th }}$ June 2016 were selected. Patients with history of any previous ocular surgery, a pterygium, corneal scarring, pre-existing astigmatism > 3.0 diopters (D), ALs $<22.0 \mathrm{~mm}$ or $>$ $27.0 \mathrm{~mm}$, previous contact lens use (within 4 weeks), severe dry eye, inflammatory disease of the eye, and/or systemic connective tissue disease were excluded. Their preoperative and post-operative records were collected and analyzed. Thirty cataractous eyes of 23 adult patients who underwent cataract surgery, with no history of any previous ocular surgery or laser, were selected. Detailed ophthalmological examinations were performed in the following order: measurement of refractive error and k-readings by using autorefractor (Huvitz HRK7000), assessment of best-corrected visual acuity (BCVA) using Snellen's acuity chart, slit lamp examination, optical biometric measurements with AL-scan, intraocular pressure (IOP) measurement using Goldmann Applanation tonometer and fundus examination using 90D lens was done.

One surgeon performed all of the cataract surgeries using a $2.75 \mathrm{~mm}$ clear corneal incision on the steep axis with implantation of the IOL in the bag. Phacoemulsification was performed on the steep corneal axis, because surgically induced astigmatism was to be minimized. Foldable IOL was implanted in the bag. The A-constants used for IOL power calculations with AL-scan for the SN60WF and MA60AC were 118.9 and 118.6 respectively. To restrict the comparison of eyes, IOL power calculations were performed only using the SRK/T formula, which is universally accepted and suitable for ALs between 22.0 and $27.0 \mathrm{~mm}$.

The postoperative final objective refraction and kreadings were measured using autorefractor (Huvitz HRK-7000) 4 weeks after cataract surgery. Subjective refraction was evaluated at the same time. Comparisons were performed in terms of objective refraction. $\mathrm{K}$ measurements of the AL-scan were compared to autorefractor data.

\section{RESULTS}

We studied 30 eyes of 23 patients (16 right and 14 left eyes) who underwent phacoemulsification. Mean age was 62.4 years with the youngest patient being 36 years old while the maximum age was 85 years. Males were $56.6 \%$ while females were $43.3 \%$. Data analysis showed that the mean post-operative spherical equivalent was plano in $53 \%$ of cases. With mean spherical equivalent -0.05 . The keratometric power was compared between autorefractor and AL-scan. The mean power by autorefractor was $44.4 \mathrm{D}$ while with AL-scan it was 44.7 D. There were no intraoperative complications or postoperative subjective complaints (such as halo or glare) in our patients.

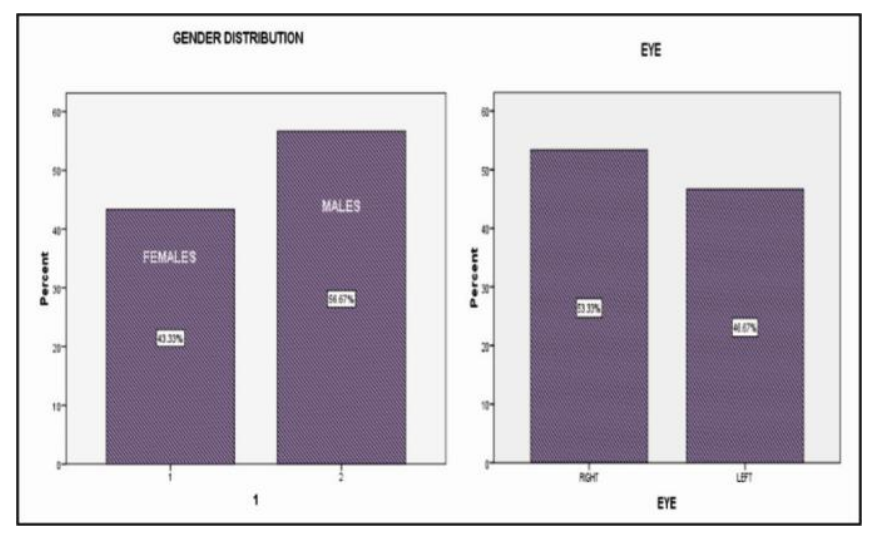

Graph 1: Gender and eye distribution

\section{DISCUSSION}

Accurate IOL power calculation is very important for attainment of patient satisfaction after cataract surgery ${ }^{9,10,11}$. Residual refractive error after the surgery is a major cause of dissatisfaction and may require IOL explantation ${ }^{9}$. The main causes of inaccuracy in biometry are $\mathrm{K}$ errors and incorrect $\mathrm{AL}$ determination $^{10}$. 
Currently, AL measurement using optical biometry is regarded to be the most accurate ${ }^{11}$. However, in cases with dense cataracts, USB is more successful ${ }^{12}$. The AL-Scan is a noncontact optical biometer using an $830 \mathrm{~nm}$ superluminescent diode as a light source. The device is very fast, measuring 6 different parameters within a few seconds: AL, corneal curvature radius, anterior chamber depth (ACD), central corneal thickness (CCT), pupil size, and whiteto-white distance (WTW). The device employs the partial coherence laser interferometry to measure AL within the range 14 to $40 \mathrm{~mm}$. The system incorporates 3-dimensional autotracking and autoshot features for ease of use in practice. The Scheimpflug principle is used for measurement of ACD and CCT. Corneal power is determined by keratometry at 2.4 and $3.3 \mathrm{~mm}$ diameter circles. The $2.4-\mathrm{mm}$ circle data are used to calculate IOL power. In USB, power is calculated by measuring the time delay of the sound wave echo received from the corneal surface and the internal limiting membrane. In contrast in optical biometry laser light is reflected from the retinal pigment epithelium ${ }^{13,14}$.

Several studies have compared the refractive outcomes yielded by earlier optical devices with those afforded by USB, mainly in the context of IOL calculations. These studies found that optical devices gave more reliable results than USB method ${ }^{15,16}$. There is a very high reproducibility between the axial length measurements by IOL Master and USB in normal eyes $(\mathrm{r}=0.985 ; \mathrm{P}=0.001)^{17}$. The AL measurements by Lenstar which is one of the earlier biometers also gives more reliable results than those of $\mathrm{USB}^{18}$.

Several studies have shown that the repeatability and reproducibility of AL-Scan was excellent in terms of all parameters, except the WTW and PD. Excluding WTW, similarity was found between the AL-Scan and IOL Master results ${ }^{19}$. Another study also found that the repeatability and reproducibility of both devices were high for all ocular biometry measurements tested (ICC $=0.87-1.00)$. Except for the WTW and corneal diameter $(\mathrm{ICC}=0.44)$, the extent of agreement between the 2 instruments was high (ICC $=0.98$ $0.99)^{20}$. The mean absolute error (MAE) in terms of IOL power prediction was $0.42 \pm 0.08 \mathrm{D}$ with the $\mathrm{AL}_{\mathrm{Scan}}{ }^{21}$.

A study undertaken previously showed that the repeatability and reproducibility of AL-Scan is excellent in terms of all parameters, except the WTW and PD and compares favorably with the IOL
Master $^{22}$. Another study also found that the repeatability and reproducibility of both devices were high for all ocular biometry measurements tested except for the WTW corneal diameter ${ }^{23}$. Both these studies also showed that the IOL power calculation was similar with the AL-scan and IOL Master.

The limitation of our study was that the small sample size but other studies done on AL-scan measurements have shown similar results making it an excellent choice for IOL calculations.

\section{CONCLUSION}

Intraocular lens power calculations done by AL-scan are easy to use, reliable and result in excellent refractive outcomes. Since minimum expertise is required to use AL-scan, technicians can easily perform it.

\section{Ethical Approval}

The study was approved by the Institutional review board/Ethical review board.

\section{Conflict of Interest}

Authors declared no conflict of interest

\section{Authors' Designation and Contribution}

Mehvash Hussain; Assistant Professor: Study design, manuscript writing, final review.

Muhammad Muneer Quraishy; Professor: Data collection, analysis, final review.

Muhammad Akram; Senior Medical Officer: Study design, manuscript writing, final review.

\section{REFERENCES}

1. Wang J-K, Hu C-Y, Chang S-W. Intraocular lens power calculation using the IOL Master and various formulas in eyes with long axial length. J Cataract Refract Surg 2008; 34 (2): 262-7.

2. Hoffer KJ. The Hoffer Q formula: a comparison of theoretic and regression formulas. J Cataract Refract Surg. 1993; 19 (6): 700-12.

3. Sanders DR, Retzlaff JA, Kraff MC, Gimbel HV, Raanan MG. Comparison of the SRK/T formula and other theoretical and regression formulas. J Cataract Refract Surg. 1990; 16 (3): 341-6.

4. Findl O, Drexler W, Menapace R, Heinzl H, Hitzenberger CK, Fercher AF. Improved prediction 
of intraocular lens power using partial coherence interferometry. J Cataract Refract Surg. 2001; 27 (6): 861-7.

5. Goel S, Chua C, Butcher M, Jones CA, Bagga P, Kotta S. Laser vs. ultrasound biometry-a study of intraand interobserver variability. Eye, 2004; 18 (5): 514-8.

6. Rose LT, Moshegov CN. Comparison of the Zeiss IOLMaster and Applanation A-scan ultrasound: biometry for intraocular lens calculation. Clin Exp Ophthalmol. 2003; 31 (2): 121-4.

7. Németh J, Fekete O, Pesztenlehrer N. Optical and ultrasound measurement of axial length and anterior chamber depth for intraocular lens power calculation. J Cataract Refract Surg. 2003; 29 (1): 85-8.

8. Tehrani M, Krummenauer F, Blom E, Dick HB. Evaluation of the practicality of optical biometry and applanation ultrasound in 253 eyes. J Cataract Ref Surg. 2003; 29 (4): 741-6.

9. Mamalis N. Complications of foldable intraocular lenses requiring explantation or secondary intervention: 1998 survey. J Cataract Refract Surg. 2000; 26 (5): 766-772.

10. Jin GJ, Crandall AS, Jones JJ. Intraocular lens exchange due to incorrect lens power. Ophthalmology, 2007; 114 (3): 417-424.

11. Chen Y-A, Hirnschall N, Findl O. Evaluation of 2 new optical biometry devices and comparison with the current gold standard biometer. J Cataract Refract Surg. 2011; 37 (3): 513-517.

12. Prinz A, Neumayer T, Buehl W, Kiss B, Sacu S, Drexler W, Findl O. Influence of severity of nuclear cataract on optical biometry. J Cataract Refract Surg. 2006; 32 (7): 1161-65.

13. Raj PS, Ilango B, Watson A. Measurement of axial length in the calculation of intraocular lens power. Eye, 1998; 12 (Pt 2): 227-9.

14. Aktas S, Aktas H, Tetikoglu M, Sagdk HM, Özcura F. Refractive Results Using a New Optical Biometry Device: Comparison With Ultrasound Biometry Data. Medicine, 2015; 94 (48): e2169.
15. Buckhurst PJ, Wolffsohn JS, Shah S, Naroo SA, Davies LN, Berrow EJ. A new optical low coherence reflectometry device for ocular biometry in cataract patients. Br J Ophthalmol. 2009; 93 (7): 949-953.

16. Jasvinder S, Khang TF, Sarinder KKS, Loo VP, Subrayan V. Agreement analysis of LENSTAR with other techniques of biometry. Eye, 2011; 25 (6): 71724.

17. Németh J, Fekete O, Pesztenlehrer N. Optical and ultrasound measurement of axial length and anterior chamber depth for intraocular lens power calculation. J Cataract Refract Surg. 2003; 29 (1): 85-8.

18. Goel S, Chua C, Butcher M, Jones CA, Bagga P, Kotta S. Laser vs. ultrasound biometry-a study of intraand interobserver variability. Eye, 2004; 18 (5): 514-8.

19. Huang J, Savini G, Li J, Lu W, Wu F, Wang J, et al. Evaluation of a new optical biometry device for measurements of ocular components and its comparison with IOL Master. Br J Ophthalmol. 2014; 98 (9): 127781.

20. Srivannaboon S, Chirapapaisan C, Chonpimai P, Koodkaew S. Comparison of ocular biometry and intraocular lens power using a new biometer and a standard biometer. J Cataract Refract Surg. 2014; 40 (5): 709-715.

21. Kaswin G, Rousseau A, Mgarrech M, Barreau E, Labetoulle M. Biometry and intraocular lens power calculation results with a new optical biometry device: comparison with the gold standard. J Cataract Refract Surg. 2014; 40 (4): 593-600.

22. Mandal P, Berrow EJ, Naroo SA. Validity and repeatability of the Aladdin ocular biometer. $\mathrm{Br} \mathrm{J}$ Ophthalmol. 2014; 98 (2): 256-258.

23. Salouti R, Nowroozzadeh MH, Zamani M, Ghoreyshi M, Khodaman MA, Comparison of Horizontal corneal diameter measurements using the Orbscan IIz and Pentacam HR systems. Cornea, 2013; 32 (11): 1460-1464. 